Nervenarzt $2021 \cdot 92: 168$

https://doi.org/10.1007/s00115-020-01054-0

Angenommen: 17. Dezember 2020

Online publiziert: 19. Januar 2021

(c) Springer Medizin Verlag GmbH, ein Teil von Springer Nature 2021



Check for
updates

C. J. Schwarzbach · A. J. Grau

Neurologische Klinik, Klinikum der Stadt Ludwigshafen am Rhein, Ludwigshafen, Deutschland

\title{
Argumente für eine strukturierte ambulante Schlaganfallnachsorge
}

\section{Erwiderung}

Zum Leserbrief von Schwarz C et al (2021) Dysphagie und spastische Bewegungsstörung nach Schlaganfall. Nervenarzt. https:// doi.org/10.1007/s00115-020-01053-1

\section{Originalbeitrag}

Schwarzbach CJ, Grau AJ (2020) Komplikationen nach Schlaganfall. Nervenarzt 91:920-925. https://doi.org/10.1007/s00115-020-00988-9

Sowohl die Komplexität als auch das weite Spektrum vaskulärer wie nichtvaskulärer Komplikationen nach einem Schlaganfall stellt den ambulanten Behandler vor besondere Herausforderungen. In ihrem Leserbrief ergänzen Schwarz et al. die Liste nichtvaskulärer Komplikationen um das hochrelevante Themenfeld der Dysphagie und geben zugleich wichtige Hinweise zur Bedeutung und zum Management der Spastizität nach einem Schlaganfall. Der Leserbrief stellt somit eine beachtenswerte Ergänzung zu unserer Übersicht dar.

Die Liste der relevanten Komplikationen ließe sich ungeachtet dessen noch erweitern. Bei der Entlassung aus der stationären Behandlung leidet ein Viertel der Schlaganfallpatienten unter einer Inkontinenz. Ein Jahr nach der Entlassung aus dem Krankenhaus sind es noch immer 15\% [1]. Neben körperlichen Leiden determinieren zudem vor allem soziale Bedürfnisse die Lebensqualität der Betroffenen und verdienen somit verstärkte Beachtung in der Schlaganfallnachsorge [2].

Aus der Relevanz und Komplexität des Themenfeldes leitet sich die Forderung nach einer strukturierten, sektorenübergreifenden und multidisziplinären ambulanten Nachsorge für Schlaganfallpatienten $\mathrm{ab}$, wie sie flächendeckend noch immer nicht etabliert ist. Eben hierfür liefert der Leserbrief von Schwarz et al. indirekt nun weitere Argumente.

\section{Korrespondenzadresse}

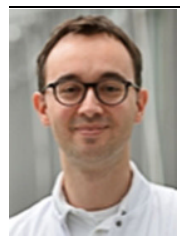

Dr. C. J. Schwarzbach Neurologische Klinik, Klinikum der Stadt Ludwigshafen am Rhein Bremserstr. 79, 67063 Ludwigshafen, Deutschland schwarch@klilu.de

Interessenkonflikt. C.J. Schwarzbach und A.J. Grau sind Mitglieder der Kommission Nachsorge der Deutschen Schlaganfall-Gesellschaft und berichten über eine projektgebundene Förderung durch den Innovationsfonds des Gemeinsamen Bundesausschusses (G-BA) mit Förderkennzeichen 01NVF17032 sowie Autorentätigkeit für den Elsevier-Verlag auf Honorarbasis.

\section{Literatur}

1. Thomas LH, Coupe J, Cross LD, Tan AL, Watkins CL (2019) Interventions for treating urinary incontinence after stroke in adults. Cochrane Database Syst Rev 2(2):CD4462

2. Hotter B, Padberg I, Liebenau A, Knispel P, Heel S, Steube D, Wissel J, Wellwood I, Meisel A (2018) Identifying unmet needs in long-term stroke care using in-depth assessment and the post-stroke checklist-the managing aftercare for stroke (MAS-I) study. Eur Stroke J3(3):237-245 